\title{
Nanomotor-based biocatalytic patterning of helical metal microstructures $\dagger$
}

Cite this: Nanoscale, 2013, 5, 1310

\author{
Kalayil Manian Manesh, Susana Campuzano, $¥$ Wei Gao, $\neq$ \\ María Jesús Lobo-Castañón,‡ Isao Shitanda, $\neq$ Kiarash Kiantaj‡ and Joseph Wang*
}

Received 5th October 2012

DOI: $10.1039 / \mathrm{c} 2 \mathrm{nr} 33040 \mathrm{~g}$

www.rsc.org/nanoscale

A new nanomotor-based surface-patterning technique based on the movement of a magnetically powered enzyme-functionalized flexible nanowire swimmer offers the ability to create complex helical metal microstructures.

There has been a considerable recent interest in synthetic nanomotors, based on different propulsion mechanisms, owing to their great promise for a wide range of future technological applications. ${ }^{1-10}$ Self-propelled nano/microscale machines have demonstrated considerable potential for performing diverse operations and important tasks, ranging from isolation of biomaterials, ${ }^{11}$ nanotool-based drilling, ${ }^{12}$ delivery of therapeutic payloads, ${ }^{13}$ to environmental remediation. ${ }^{\mathbf{1 4}}$

This communication reports on the use of flexible magnetic nanowire swimmers for biocatalytic patterning of complex surface microstructures. The preparation of well-defined surface micro/ nanostructures represents an important goal of microfabrication. Tip-based scanning-probe (SP) techniques have been extremely useful for depositing chemical or biological materials onto flat substrates. ${ }^{15}$ Such tip-based SP fabrication methods commonly rely on the controlled movement of a functionalized tip along predetermined paths for a localized surface modification. Willner et al. ${ }^{16-19}$ have demonstrated the successful combination of Dip-PenNanolithography (DPN) and biocatalytic inks for generating Au or $\mathrm{Ag}$ nanowires in connection with the deposition of glucose oxidase (GOx) or alkaline-phosphatase lines, respectively. However, patterning of three-dimensional structures with high topological complexity of a helix represents a major fabrication challenge even with advanced lithographic techniques such as DPN. ${ }^{16-19}$

Recent advances in nanomotors have facilitated the realization of the new nanomotor-based direct biocatalytic patterning method.

Department of Nanoengineering, University of California San Diego, La Jolla, CA 92093, USA. E-mail: josephwang@ucsd.edu; Fax: +1 (858) 534-9553; Tel: +1 (858) 246-0128

† Electronic supplementary information (ESI) available: Supporting figures, videos and details of enzymatic activity studies. See DOI: 10.1039/c2nr33040g

† S. C., W. G., M. J. L. -C., K. K. and I. S. contributed equally to this work.
These include the ability to navigate the motors along predetermined complex paths, to control and regulate their speed, to functionalize them with different biological or chemical entities, and to move them rapidly over large areas. ${ }^{20,21}$ Previous studies reported that HRP-functionalized catalytic nanowire motors can be used for writing localized polymeric lines. ${ }^{22}$ However, the limited propulsion of these catalytic nanomotors in high-ionic strength environments and the peroxide-fuel requirement hinder the applications of these nanomotors. ${ }^{23}$ Similar to different tip-based SP fabrication techniques, ${ }^{15}$ nanomotors based on different propulsion and guidance mechanisms could be employed for creating a variety of surface microstructures. Various microstructures, made of different materials (polymers, metals, etc.), can thus be fabricated on conducting and insulating substrates based on a judicious choice of the reactants and the specific reaction involved. In view of the 'large' (submicrometer) size of catalytic nanomotors, compared to common SP tips, the new nanomotor 'writing' method is currently limited to the creation of microscale surface features.

This study illustrates for the first time the creation of complex helical metallic microscale surface structures based on local reaction of enzyme-functionalized artificial fuel-free magnetic nanomotors. Magnetically controlled motion, inspired by the motility of microorganisms, represents an attractive route for addressing the challenge of nanoscale propulsion, as they can perform complex maneuvers while obviating fuel requirements. ${ }^{24-26}$ Highly efficient flexible magnetic nanowire swimmers have been prepared recently by a simple membrane-template electrodeposition route. ${ }^{27-29}$ The resulting magnetic nanowire swimmers have already been used successfully for the directed delivery of drug-loaded microparticles to HeLa cancer cells in the cell culture media. ${ }^{30}$

In the following sections we will illustrate biocatalytically induced growth of distinct helical Au microstructures during the magnetically powered movement of GOx-functionalized flexible nanowire motors. The patterning of helical surface microstructures commonly represents a major challenge using conventional nanoand microfabrication methods. As illustrated in Scheme 1, the new nanomotor-induced surface 'writing' protocol relies on the controlled movement of an enzyme-functionalized nanomotor 


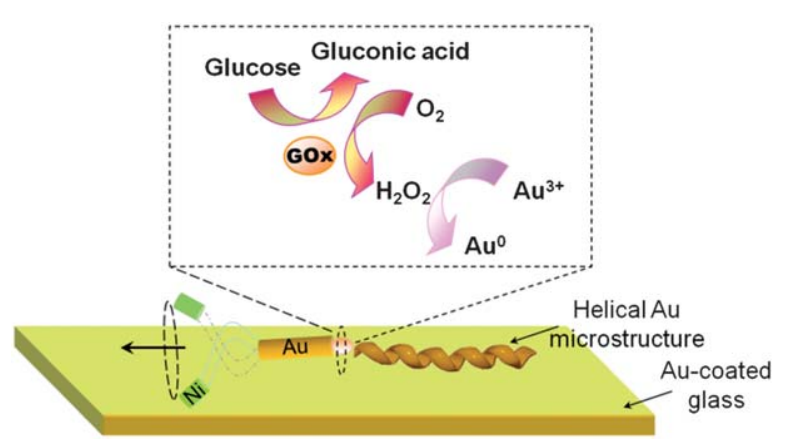

Scheme 1 Schematic representation of the nanomotor-induced biocatalytic metallization of a helical Au microstructure using a GOx-Au/Agflex $/ \mathrm{Ni}$ magnetic nanowire swimmer. Inset shows the biocatalytic reactions involved in the $\mathrm{Au}$ patterning.

along a localized surface and leads to distinct helical structures due to reproducible rotation of the immobilized biocatalytic layer. This is accomplished by using magnetically driven GOx-functionalized flexible three-segment $\mathrm{Au} / \mathrm{Ag}_{\text {flex }} / \mathrm{Ni}$ nanowire motors under a rotating magnetic field. Previously we demonstrated that such magnetically powered nanowire motors, containing a flexible silver joint, can swim efficiently under an external rotating magnetic field. $^{27}$

As illustrated in Scheme 1 our new surface 'writing' concept relies on the confinement of GOx onto the $\mathrm{Au}$ end of a flexible $\mathrm{Au} /$ $\mathrm{Ag}_{\text {flex }} / \mathrm{Ni}$ magnetic $5.5 \mu \mathrm{m}$ long nanowire motor for localized biometallization through a $\mathrm{H}_{2} \mathrm{O}_{2}$-mediated deposition of helical $\mathrm{Au}$ microwires in the presence of glucose and $\mathrm{AuCl}_{4}{ }^{-}$ions. The enzyme confinement onto the end of the Au segment was accomplished by covalent immobilization using carbodiimide/succinimide onto a carboxylic acid terminated alkanethiol thiolated self-assembled monolayer (SAM) (see Fig. S1 in the ESI $\dagger$ ). Under a rotating magnetic field, the $\mathrm{Ni}$ segment of the flexible $\mathrm{Au} / \mathrm{Ag}_{\text {flex }} / \mathrm{Ni}$ nanomotors generates a cone-shaped rotation which causes rotation of the $\mathrm{Au}$ segment on the opposite end..$^{27}$ Defined surface microstructures can thus be patterned through a controlled movement and accurate positioning of the GOx-modified nanomotor, in a manner analogous to the AFM biocatalytic lithography and $\mathrm{H}_{2} \mathrm{O}_{2}$-mediated growth of Au microstructures. ${ }^{17,18}$ Video 1 (in the ESI $\dagger$ ) displays the movement of a flexible $\mathrm{GOx}-\mathrm{Au} / \mathrm{Ag}_{\text {flex }} / \mathrm{Ni}$ nanowire in a solution mixture containing $10 \mathrm{mM}$ glucose and $0.3 \mathrm{mM} \mathrm{AuCl}_{4}{ }^{-}$. During the rotation of the GOx-functionalized $\mathrm{Au}$ segment, the biocatalytic oxidation of glucose leads to the formation of distinct helical plumes of the $\mathrm{H}_{2} \mathrm{O}_{2}$ product (eqn (1)). The latter acts as a reducing agent for the localized catalytic reduction of $\mathrm{AuCl}_{4}{ }^{-}$ions on a Aucoated glass (eqn (2)), leading to unique surface patterns of helical $\mathrm{Au}$ microstructures that reflect the rotation of the enzyme and the corresponding peroxide plume. AuNP seeds were shown to be essential for biocatalytically induced growth of $\mathrm{Au}$ nanowires on glucose oxidase. ${ }^{18}$ Magnetically guided motion of the GOx-modified motor thus leads to a localized biocatalytic metallization and hence to the direct 'writing' of $\mathrm{Au}$ microfeatures in the presence of GOx along the nanomotor path. It is expected that the modification of such magnetic swimmers with different biocatalysts will lead to motor-based patterning of different metal microstructures.

$$
\begin{gathered}
\text { Glucose }+\mathrm{O}_{2} \rightarrow \text { gluconic acid }+\mathrm{H}_{2} \mathrm{O}_{2} \\
\mathrm{AuCl}_{4}{ }^{-}+3 / 2 \mathrm{H}_{2} \mathrm{O}_{2} \stackrel{\mathrm{Au}(\text { surf })}{\longrightarrow} \mathrm{Au}^{0}+4 \mathrm{Cl}^{-}+3 \mathrm{H}^{+}+3 / 2 \mathrm{O}_{2}
\end{gathered}
$$

Fig. 1A illustrates the microscale Au helical surface microstructure created by the biocatalytic metallization reaction using a GOx$\mathrm{Au} / \mathrm{Ag}_{\text {flex }} / \mathrm{Ni}$ nanowire. The biometallization reaction was carried out in $10 \mathrm{mM}$ glucose and $0.3 \mathrm{mM} \mathrm{AuCl}_{4}{ }^{-}$at a rotation frequency of 7.5 Hz. The controlled translational movement of the swimmer, including the defined rotation of the GOx-modified Au segment, thus results in distinct $\mathrm{Au}$ helical surface microstructures. In this specific case, a $\sim 500 \mathrm{~nm}$ wide ( $\sim 15 \mu \mathrm{m}$ long) wire with an average height of $\sim 500 \mathrm{~nm}$, is obtained (Fig. 1A). In order to confirm that the patterning is solely due to the biocatalytic process, we performed control experiments with SAM-modified flexible $\mathrm{Au} / \mathrm{Ag}_{\text {flex }} / \mathrm{Ni}$ nanowires without GOx. As expected, no such microstructure was observed in the absence of the enzyme (Fig. 1B), clearly demonstrating the biocatalytic nature of the patterning process. Similarly, no surface microstructures were observed in additional control experiments without the glucose substrate or the $\mathrm{AuCl}_{4}{ }^{-}$ions in the developing solution, or using a bare glass surface without the AuNP seeds (not shown). All of these control experiments indicate clearly that the localized biocatalytic formation of $\mathrm{H}_{2} \mathrm{O}_{2}$ is essential to induce the growth of the microstructure and corroborate that the AuNP seeds on the glass surface act as catalysts for the reduction of $\mathrm{AuCl}_{4}{ }^{-}$by $\mathrm{H}_{2} \mathrm{O}_{2}{ }^{16}$ The formation of the helical Au patterning was also confirmed using the energy-dispersive X-ray (EDX) elemental mapping measurements shown in Fig. 2.

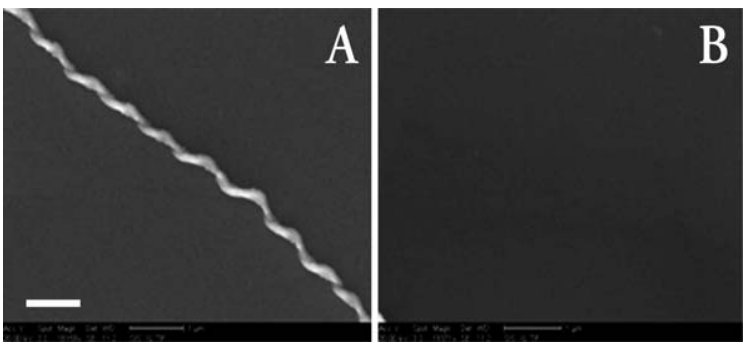

Fig. 1 SEM images of a biocatalytically generated helical Au micropattern over a Au-coated glass surface using an $\mathrm{Au} / \mathrm{Ag}_{\text {flex }} / \mathrm{Ni}$ magnetic microswimmer modified (A) and unmodified (B) with GOx on the Au side. The biometallization reaction was carried out in a solution containing $10 \mathrm{mM}$ glucose and $0.3 \mathrm{mM} \mathrm{AuCl}_{4}{ }^{-}$ Magnetic nanomotors rotation frequency: $7.5 \mathrm{~Hz}$. Scale bar, $1 \mu \mathrm{m}$.
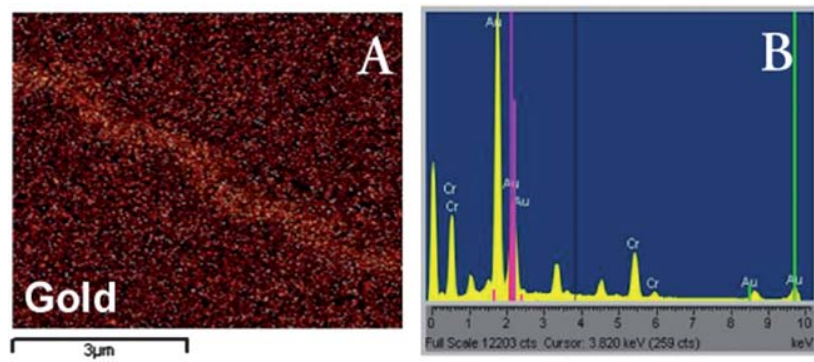

Fig. 2 Elemental analysis of Au helical patterning: Au (A) and EDX elemental mapping (B) 
Our results also confirm the enzymatic activity of the GOx attached to the flexible $\mathrm{Au} / \mathrm{Ag}_{\text {flex }} / \mathrm{Ni}$ nanowires, despite the harsh alkaline conditions used for dissolving the alumina nanowires template (described in detail in Section Bio-functionalization of the nanowires). This was supported by additional electrochemical experiments that were carried out in order to estimate the enzymatic activity after exposure to these harsh membrane-dissolution conditions. The results of these experiments, described in the ESI $\dagger$ (Section Residual GOx enzymatic activity after membrane dissolution), demonstrate that the attached enzyme retains approximately the $23.5 \%$ of its original activity. This is supported by early observations of Martin and coworkers who reported that GOx nanotubes - prepared by a similar protocol - are able to catalyze the oxidation of glucose. ${ }^{31}$

Most favourable results were observed using $10 \mathrm{mM}$ glucose, $0.3 \mathrm{mM} \mathrm{AuCl}_{4}{ }^{-}$and following an initial $30 \mathrm{~min}$ incubation time. No apparent patterning was noticeable using incubation times shorter than 15 min or using glucose concentrations lower than $1 \mathrm{mM}$. These results could be attributed to the requirement of a threshold $\mathrm{H}_{2} \mathrm{O}_{2}$ level for the $\mathrm{Au}$ enlargement reaction to take place. ${ }^{17}$ In view of these results, the height and width of the resulting helical microstructures appear to depend on the biocatalytic "development" time-interval ${ }^{17}$ and the concentration of glucose. The resulting structural features can thus be tuned by a rational adjustment of these parameters, reflecting the mechanistic aspects involved in the helical biometallization patterning. Obviously, the resulting helical surface patterns are extremely unique. Creating such three dimensional surface patterning is commonly very challenging even by using state-of-the-art lithographic techniques.

Controlled nanomotor motion along predetermined paths, with a fine control over its position and rotation, is essential for generating reproducible predefined surface patterning. In particular, a judicious control of the magnetic actuation allows the fine tuning and tailoring of the resulting microstructures. For example, as clearly illustrated in Fig. 3, the period and width of the helical structure can be controlled by using different rotation frequencies (7.5, 12 and $15 \mathrm{~Hz}, \mathrm{~A}-\mathrm{C}$ ). Using the lower frequency (Fig. 3A) the diameter of the helical structure created is less than $500 \mathrm{~nm}$; the line width increases upon raising the frequency to $12 \mathrm{~Hz}$ (Fig. 3B). However, the highest frequency of $15 \mathrm{~Hz}$ resulted in microscale lines instead of a helical microstructure (Fig. 3C). A simple calculation using frequency and speed of the nanomotors indicates that the expected pitch of the helical structure (the width of one complete helix turn), when the frequency of the rotation is between 7.5 and $12 \mathrm{~Hz}$, should be both around $1 \mu \mathrm{m}$ due to the nearly linear relation between the frequency and speed. ${ }^{28}$ Our experimental results also

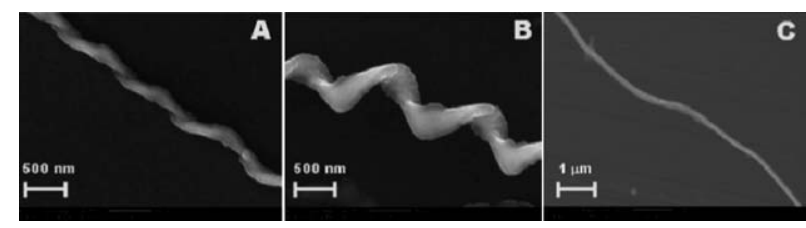

Fig. 3 SEM images of the biocatalytically generated helical Au micropatterns obtained using GOx-modified $\mathrm{Au} / \mathrm{Ag}_{\mathrm{flex}} / \mathrm{Ni}$ magnetic swimmers at different rotation frequencies: 7.5 (A), 12 (B) and $15 \mathrm{~Hz}(\mathrm{C})$. confirm that in both cases the pitch of the metallic helical structures is around $1 \mu \mathrm{m}$.

As illustrated in Fig. 3, the lower rotation frequency $(7.5 \mathrm{~Hz})$ induced a lower diameter of the helical structure. However, the exact rotation radius of the Au segment highly depends upon the elasticity and length of the flexible Ag joint. Accordingly, it is difficult to predict the exact correlation between the radius of the rotation of the motors and the width of the helical structures.

Our experimental data proves that there is a cut-off frequency around $15 \mathrm{~Hz}$ at which nanomotors stop to respond to the rotation frequency of the external magnets. No obvious helical structures have been formed over $15 \mathrm{~Hz}$. Instead, a fairly straight Au line forms and deposits on the surface. This can be attributed to the fact that at this frequency the speed of the nanomotors will increase to $12 \mu \mathrm{m}$ $\mathrm{s}^{-1}$, leading to short contact times of GOx with the Au surface and to a different plume dynamics of the $\mathrm{H}_{2} \mathrm{O}_{2}$ product, and hence to the absence of helical surface patterns.

Another important advantage of this new methodology, compared to DPN-based biocatalytic approaches, is that the developed nanomotor-based approach is not a self-limiting process. The nanomotor-induced surface 'writing' strategy presented the biocatalytic reaction and hence the patterning does not come to a standstill due to the physical blocking of the enzyme observed in the biocatalytic-DPN method. ${ }^{17,19}$ Another distinct feature of the present nanomotor-based nanofabrication strategy is that it can be readily used to prepare defined structures of finite lengths. Since the pattern is formed only when the nanomotor navigates in close proximity to the Au-coated glass surface, one can terminate the process by guiding the motor to an upper plane, thus enabling the "writing" of finite length microstructures.

\section{Conclusions}

In conclusion, we presented a new and attractive nanomotor-based biocatalytic surface patterning route approach for generating threedimensional $\mathrm{Au}$ microstructures with the high topological complexity (e.g., helical shape) using enzyme modified magnetic nanomotors. This method merges the fine control over the position and translational motion of the magnetic nanowires, essential for generating reproducible predefined patterns, with the versatility of biocatalytic metal deposition reactions. Although the present study has demonstrated for the first time the use of magnetic nanowire motors along with the "biocatalytic inks" as a new paradigm for generating metallic helical nanostructures the concept could be easily extended to other "biocatalytic inks" that generate conducting, semiconducting and insulating polymers, as well as microscale magnetic or semiconductor wires. Future effort will also aim at gaining better understanding of the dynamics, dispersion and directionality of the plume of the peroxide product (in relation to the swimmer actuation) towards enhanced control of the patterning process. The ability to grow tailored metallic helical patterns and the application of different biocatalytic reactions pave the way for the generation of a variety of complex metal microstructures. A judicious selection of the experimental variables allows tuning of the exact writing patterns. The new nanomotor-based biocatalytic patterning thus holds a considerable promise for generating different surface microstructures of different materials and shapes 
in connection with diverse (bio)chemical reactions, towards the creation of complex surface circuitries.

\section{Experimental section}

\section{Reagents and solutions}

11-Mercaptoundecanoic acid (MUA), $N$-hydroxysuccinimide (NHS), 1-(3-dimethylaminopropyl)- $N N^{\prime}$-ethylcarbodiimide hydrochloride (EDC) were purchased from Sigma-Aldrich. Glucose oxidase (GOx, from Aspergillus niger, EC 1.1.3.4), horseradish peroxidase (HRP, Type II from Horseradish, EC 1.11.1.7), glucose, cupric sulfate pentahydrate, nickel(II) chloride hexahydrate, nickel(II) sulfamate tetrahydrate, boric acid and glucose were purchased from Sigma. The HRP substrate 3,3',5,5'-tetramethylbenzidine (TMB, Neogen K-blue enhanced activity substrate, containing $\mathrm{H}_{2} \mathrm{O}_{2}$ ) was obtained from Neogen (Lexington, KY). Reagents were used without any further purification. Experiments were carried out at room temperature. $\mathrm{Au}$ (Orotemp 24 RTU RACK) and Ag (1025 RTU @ 4.5 Troy/gallon) plating solutions were purchased from Technic Inc., Anaheim, CA.

Another solution employed, prepared in deionized water, was: $0.1 \mathrm{M}$ phosphate buffer, $\mathrm{pH}$ 7.4. All chemicals used were of analytical-reagent grade, and deionized water was obtained from a Millipore Milli-Q purification system (18.2 $\mathrm{M} \Omega \mathrm{cm})$.

\section{Apparatus}

Electrochemical deposition of nanowires and chronoamperometric measurements were carried out with a $\mathrm{CHI} 621 \mathrm{~A}$ potentiostat $(\mathrm{CH}$ Instruments, Austin, TX). Scanning electron microscopy (SEM) images were obtained with a Phillips XL30 ESEM instrument using an acceleration potential of $20 \mathrm{kV}$. An optical microscope (Nikon Instrument Inc. Ti-S/L100), coupled with a $40 \times$ objective, was used for the visualization.

To evaluate the residual enzymatic activity remained after membrane dissolution, commercial screen printed rough Au electrodes (220AT, $\phi \sim 4 \mathrm{~mm}, \mathrm{Au} / \mathrm{SPEs}$ ) purchased from Dropsens were used.

\section{Synthesis of the flexible $\mathrm{Au} / \mathrm{Ag}_{\text {flex }} / \mathrm{Ni}$ magnetic nanowires}

$\mathrm{The} \mathrm{Au} / \mathrm{Ag}_{\text {flex }} / \mathrm{Ni}$ magnetic nanowires were prepared using templatedirected electrodeposition protocols. A silver film was first sputtered on one side of the porous alumina membrane template containing $200 \mathrm{~nm}$ diameter cylindrical pores and $25 \mathrm{~mm}$ diameter (Catalog no. 6809-6022; Whatman, Maidstone, UK) to serve as a working electrode. The membrane was then assembled in a plating cell with an aluminum foil serving as a contact for the sputtered silver. Copper was electrodeposited in the branch area from a $1 \mathrm{M}$ $\mathrm{CuSO}_{4} \cdot 5 \mathrm{H}_{2} \mathrm{O}$ solution, using a charge of $10 \mathrm{C}$ and a potential of $-1.0 \mathrm{~V}(v s$. Ag/AgCl), along with a Pt-wire counter electrode; subsequently, nickel was plated from a nickel plating solution containing $20 \mathrm{~g} \mathrm{~L}^{-1} \mathrm{NiCl}_{2} \cdot 6 \mathrm{H}_{2} \mathrm{O}, 515 \mathrm{~g} \mathrm{~L}^{-1} \mathrm{Ni}\left(\mathrm{H}_{2} \mathrm{NSO}_{3}\right)_{2} \cdot 4 \mathrm{H}_{2} \mathrm{O}$, and $20 \mathrm{~g} \mathrm{~L}^{-1}$ $\mathrm{H}_{3} \mathrm{BO}_{3}$ at $-1.0 \mathrm{~V}$ (vs. $\mathrm{Ag} / \mathrm{AgCl}$ ) for $3 \mathrm{C}$; silver was plated at $-0.9 \mathrm{~V}(v s$. $\mathrm{Ag} / \mathrm{AgCl}$ ) for a total charge of $3.5 \mathrm{C}$ using a commercial silver plating solution (1025 RTU @ 4.5 Troy/gallon; Technic Inc., Anaheim, CA); finally, Au was plated from the commercial gold-plating solution at $-1.0 \mathrm{~V}(v \mathrm{~s}$. Ag/ $\mathrm{AgCl})$, using a charge of $3 \mathrm{C}$. The sputtered silver layer and copper sacrificial layer were mechanically removed from the membrane by polishing with 3-4 $\mu \mathrm{m}$ alumina powder. The sacrificial copper layer was dissolved using a $20 \% \mathrm{HCl}$ solution containing $0.5 \mathrm{M} \mathrm{CuCl}_{2}$. The membrane was then dissolved in a $\mathrm{NaOH}(3 \mathrm{M})$ solution for $30 \mathrm{~min}$ to completely release the nanowires. The nanowires were collected by centrifugation at $6000 \mathrm{rpm}$ for $5 \mathrm{~min}$ and were washed repeatedly with nanopure water $(18.2 \mathrm{M} \Omega \mathrm{cm})$ until a neutral $\mathrm{pH}$ was achieved. All non-modified nanowire solutions were stored in nanopure water at room temperature. Flexibility of the silver segment was achieved by its partial dissolution accomplished by mixing the diluted $\mathrm{Ni}-\mathrm{Ag}$-Au nanowire solution $(10 \mu \mathrm{L})$ with the $\mathrm{H}_{2} \mathrm{O}_{2}$ solution $(10 \%(\mathrm{w} / \mathrm{v}), 10 \mu \mathrm{L})$ for $1 \mathrm{~min}$. The nanowires were then washed on the glass slides using nanopure water $(18.2 \mathrm{M} \Omega \mathrm{cm})$ until a neutral $\mathrm{pH}$ was achieved.

\section{Bio-functionalization of the nanowires}

Fig. S1 in the ESI $\dagger$ depicts a schematic representation of the biofunctionalization process by exposing the Au ends of the nanowires (while in the membrane) to a $2.5 \mathrm{mM}$ ethanolic solution of MUA overnight at room temperature in order to form a dense carboxylic acid-terminated SAM. The MUA-modified nanowires (still in the membrane) were subsequently treated with a solution of NHS $(100 \mathrm{mM})$ and $\mathrm{EDC}(400 \mathrm{mM})$ in ultrapure water for $2 \mathrm{~h}$ following by

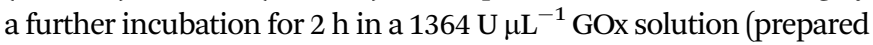
in $0.1 \mathrm{M}$ phosphate buffer solution of $\mathrm{pH}$ 7.4). Finally, the membrane with the GOx-modified nanowires was washed 3 times with ultrapure water. A diluted $\mathrm{NaOH}(0.3 \mathrm{M})$ solution was used to dissolve the membrane and release the enzyme-functionalized nanowires which were washed thoroughly with ultra pure water by repeated centrifugation at $6000 \mathrm{rpm}$ for $5 \mathrm{~min}$. These GOx-functionalized nanowires were washed repeatedly with nanopure water $(18.2 \mathrm{M} \Omega \mathrm{cm})$ until a neutral $\mathrm{pH}$ was achieved and were stored in phosphate buffer (pH 7.4) at $4{ }^{\circ} \mathrm{C}$ until their use.

\section{Magnetic driven movement}

The magnetic field was achieved by a triaxial Helmholtz coil, consisting of a homogeneous rotating magnetic field and a constant magnetic field which is perpendicular to the axis of the rotating one. ${ }^{29}$ The magnetic induction was measured using a Gaussmeter (Model 475 DSP Gaussmeter, Lake Shore Cryotronics, Inc, Westerville, $\mathrm{OH}$ ). The magnetic nanomotors were driven by a magnetic field with an unsteady component of amplitude $H_{1}$, rotating sinusoidally in a plane perpendicular to a constant component, $H_{0}$. In the experiments, $H_{1}=H_{0}=5$ Gauss.

\section{Biocatalytic patterning of helical Au microstructures using GOx- modified magnetic nanoswimmers}

The flexible thinner joint is prepared by partial dissolution of the silver segment accomplished by mixing $10 \mu \mathrm{L}$ of the diluted magnetic swimmer suspension with $10 \mu \mathrm{L}$ of a $10 \%(w / w) \mathrm{H}_{2} \mathrm{O}_{2}$ solution for $1 \mathrm{~min}$. The nanowires were then washed under the optical microscope using nanopure water $(18.2 \mathrm{M} \Omega \mathrm{cm})$ until a neutral $\mathrm{pH}$ was achieved. These thorough washings ensured complete removal of $\mathrm{H}_{2} \mathrm{O}_{2}$ used for the $\mathrm{Ag}$ partial dissolution. A 10 $\mu \mathrm{L}$ aliquot of freshly prepared GOx-functionalized flexible magnetic nanowire motors was mixed with the same volume of the developing solution (containing $10 \mathrm{mM}$ glucose and $0.3 \mathrm{mM} \mathrm{AuCl}_{4}^{-}$). 


\section{Acknowledgements}

This work was supported by DTRA Grant HDTRA1-13-1-0002. W.G. is a HHMI International Student Research fellow. S. C., M. J. L. -C. and I. S. acknowledge fellowships from Programa Becas Complutense del Amo (2010-2011), University of Oviedo and Spanish Ministerio de Ciencia e Innovacion (PR 2009-0430), and the International Scholarship Foundation of Tokyo University of Science (2010-2011), respectively.

\section{Notes and references}

1 T. E. Mallouk and A. Sen, Sci. Am., 2009, 5, 72.

2 T. Mirkovic, N. S. Zacharia, G. D. Scholes and G. A. Ozin, ACS Nano, 2010, 4, 1782.

3 J. Wang, ACS Nano, 2009, 3, 4.

4 S. Sengupta, M. E. Ibele and A. Sen, Angew. Chem., Int. Ed., 2012, 51, 8434.

5 Y. F. Mei, A. A. Solovev, S. Sanchez and O. G. Schmidt, Chem. Soc. Rev., 2011, 40, 2109.

6 S. Sanchez and M. Pumera, Chem.-Asian J., 2009, 4, 1402.

7 J. Wang and W. Gao, ACS Nano, 2012, 6, 5745.

8 M. Pumera, Nanoscale, 2010, 2, 1643.

9 A. A. Solovev, S. Sanchez, M. Pumera, Y. F. Mei and O. G. Schmidt, Adv. Funct. Mater., 2010, 20, 2430.

10 G. Loget and A. Kuhn, Nat. Commun., 2011, 2, 535.

11 S. Campuzano, J. Orozco, D. Kagan, M. Guix, W. Gao, S. Sattayasamitsathit, J. C. Claussen, A. Merkoçi and J. Wang, Nano Lett., 2012, 12, 396.

12 A. A. Solovev, W. Xi, D. H. Gracias, S. M. Harazim, C. Deneke, S. Sanchez and O. G. Schmidt, ACS Nano, 2012, 6, 1751.

13 D. Kagan, R. Laocharoensuk, M. Zimmerman, C. Clawson, S. Balasubramanian, D. Kang, D. Bishop, S. Sattayasamitsathit, L. Zhang and J. Wang, Small, 2010, 6, 2741.
14 M. Guix, J. Orozco, M. García, W. Gao, S. Sattayasamitsathit, A. A. Merkoçi, A. Escarpa and J. Wang, ACS Nano, 2012, 6, 4445.

15 H. T. Soh, K. W. Guarini and C. F. Quate, Scanning Probe Lithography, Kluwer Academic Publishers, Norwell, 2001.

16 M. Zayats, R. Baron, I. Popov and I. Willner, Nano Lett., 2005, $5,21$.

17 B. Basnar, Y. Weizmann, Z. Cheglakov and I. Willner, Adv. Mater., 2006, 18, 713.

18 I. Willner, R. Baron and B. Willner, Adv. Mater., 2006, 18, 1109.

19 B. Basnar and I. Willner, Small, 2009, 5, 28.

20 J. Wang and K. M. Manesh, Small, 2010, 6, 338.

21 S. Campuzano, D. Kagan, J. Orozco and J. Wang, Analyst, 2011, 136, 4621.

22 K. M. Manesh, S. Balasubramanian and J. Wang, Chem. Commun., 2010, 46, 5704.

23 W. F. Paxton, P. T. Baker, T. R. Kline, Y. Wang, T. E. Mallouk and A. Sen, J. Am. Chem. Soc., 2006, 128, 14881.

24 L. Zhang, J. J. Abbott, L. X. Dong, B. E. Kratochvil, D. Bell and B. J. Nelson, Appl. Phys. Lett., 2009, 94, 64107.

25 A. Ghosh and P. Fischer, Nano Lett., 2009, 9, 2243.

26 P. Tierno, R. Golestanian, I. Pagonabarraga and F. Sagues, Phys. Rev. Lett., 2008, 101, 218304.

27 W. Gao, S. Sattayasamitsathit, K. M. Manesh, D. Weihs and J. Wang, J. Am. Chem. Soc., 2010, 132, 14403.

28 O. S. Pak, W. Gao, J. Wang and E. Lauga, Soft Matter, 2011, 7, 8169.

29 W. Gao, K. M. Manesh, J. Hua, S. Sattayasamitsathit and J. Wang, Small, 2011, 7, 2047.

30 W. Gao, D. Kagan, O. S. Pak, C. Clawson, S. Campuzano, E. Chuluun-Erdene, E. Shipton, E. E. Fullerton, L. Zhang, E. Lauga and J. Wang, Small, 2012, 8, 460.

31 S. F. Hou, J. H. Wang and C. R. Martin, Nano Lett., 2005, 5, 231. 